\title{
ATMOSPHERIC MUON FLUX AT PEV ENERGIES
}

\author{
S. I. SINEGOVSKY, A. A. KOCHANOV, T. S. SINEGOVSKAYA, \\ Irkutsk State University, Gagarin Blvd 20, Irkutsk, RU-664003 Russia \\ sinegovsky@api.isu.ru \\ A. MISAKI, \\ Research Institute for Science and Technology, Waseda U., Tokyo, 169-8555, Japan \\ Innovative Research Organization, Saitama U., Saitama, Japan \\ N. TAKAHASHI \\ Graduate School of Science and Technology, Hirosaki U., Hirosaki 036-8561, Japan
}

\begin{abstract}
In the near future the energy region above few hundreds of $\mathrm{TeV}$ may really be accessible for measurements of the atmospheric muon spectrum with IceCube array. Therefore one expects that muon flux uncertainties above $50 \mathrm{TeV}$, related to a poor knowledge of charm production cross sections and insufficiently examined primary spectra and composition, will be diminished. We give predictions for the very high-energy muon spectrum at sea level, obtained with the three hadronic interaction models, taking into account also the muon contribution due to decays of the charmed hadrons.
\end{abstract}

Keywords: cosmic ray muons, high-energy hadronic interactions

PACS numbers: 95.85.Ry, 13.85.Tp

\section{Introduction}

The atmospheric muon flux as well as muon neutrino flux at high energies are inevitably dominated by the prompt component due to decays of the charmed hadrons $\left(D^{ \pm}, D^{0}, \bar{D}^{0}, D_{s}^{ \pm} \Lambda_{c}^{+}, \ldots\right)$, hence the prompt neutrino flux becomes the major source of the background in the search for a diffuse astrophysical neutrino flux 1 - 4 . Insufficiently explored processes of the charm production give rise to most uncertainty in the muon and neutrino fluxes. IceCube, the first to begin operating as the $\mathrm{km} 3$ neutrino telescope, has the real capability ${ }_{5 \mid 6}$ to measure the atmospheric muon spectrum at energies up to $1 \mathrm{PeV}$ and to shed light on the feasible range of the cross sections for the charmed particle production.

Besides, an ambiguity in high-energy behaviour of pion and kaon production cross sections affects essentially the atmospheric muon (neutrino) flux. Recent calculations 7 reveal differences (up to factor 1.8 at $10 \mathrm{PeV}$ ) in the neutrino flux because of uncertain description of the hadronic proceses involving light quarks at high energies. 
In this work we extend to higher energies the conventional muon flux calculations basing on the known hadronic interaction models with usage reliable data of the primary cosmic ray measurements. We present results of the conventional muon flux calculations in the energy range $10^{5}-10^{8} \mathrm{GeV}$ using hadronic models QGSJETIl ${ }^{89}$, SIBYLL 2.1 1011 , EPOS ${ }^{1213}$ as well as the model by Kimel and Mokhov 14 $(\mathrm{KM})$, that were tested also in recent atmospheric muon flux calculations 1516 . In order to compare the uncetainity of the conventional muon flux and prompt one we plot the prompt muon contrubition originating from decays of the charmed hadrons produced in collisions of cosmic rays with nuclei of air (for review see e.g. Refs. 1722).

\section{The method}

The high-energy muon fluxes are calculated using the approach 23 to solve the atmospheric hadron cascade equations taking into account non-scaling behavior of inclusive particle production cross-sections, rise of total inelastic hadron-nuclei crosssections, and the non-power law primary spectrum (see also Ref. 16).

To obtain the differential energy spectra of protons $p(E, h)$ and neutrons $n(E, h)$ at the atmosphere depth $h$ one needs to solve the set of equations:

$$
\frac{\partial N^{ \pm}(E, h)}{\partial h}=-\frac{N^{ \pm}(E, h)}{\lambda_{N}(E)}+\frac{1}{\lambda_{N}(E)} \int_{0}^{1} \Phi_{N N}^{ \pm}(E, x) N^{ \pm}(E / x, h) \frac{d x}{x^{2}}
$$

where $N^{ \pm}(E, h)=p(E, h) \pm n(E, h)$,

$$
\Phi_{N N}^{ \pm}(E, x)=\frac{E}{\sigma_{p A}^{i n}(E)}\left[\frac{d \sigma_{p p}\left(E_{0}, E\right)}{d E} \pm \frac{d \sigma_{p n}\left(E_{0}, E\right)}{d E}\right]_{E_{0}=E / x},
$$

$\lambda_{N}(E)=1 /\left[N_{0} \sigma_{p A}^{i n}(E)\right]$ is the nucleon interaction length in the atmosphere, $x=$ $E / E_{0}$ is the fraction of the primary nucleon energy $E_{0}$ carried away by the secondary nucleon, $d \sigma_{a b} / d E$ is the cross sections for inclusive reaction $a+A \rightarrow b+X$. The boundary conditions for Eq. (1) are $N^{ \pm}(E, 0)=p_{0}(E) \pm n_{0}(E)$.

Suppose that the solution of the system is

$$
N^{ \pm}(E, h)=N^{ \pm}(E, 0) \exp \left[-\frac{h\left(1-Z_{N N}^{ \pm}(E, h)\right)}{\lambda_{N}(E)}\right]
$$

where $Z_{N N}^{ \pm}(E, h)$ are unknown functions. Substituting Eq. (2) into Eq. (1) we find the equation for these functions $Z_{N N}^{ \pm}$(Z-factors):

$$
\frac{\partial\left(h Z_{N N}^{ \pm}\right)}{\partial h}=\int_{0}^{1} \Phi_{N N}^{ \pm}(E, x) \eta_{N N}^{ \pm}(E, x) \exp \left[-h D_{N N}^{ \pm}(E, x, h)\right] d x
$$

where $\eta_{N N}^{ \pm}(E, x)=x^{-2} N^{ \pm}(E / x, 0) / N^{ \pm}(E, 0)$,

$$
D_{N N}^{ \pm}(x, E, h)=\frac{1-Z_{N N}^{ \pm}(E / x, h)}{\lambda_{N}(E / x)}-\frac{1-Z_{N N}^{ \pm}(E, h)}{\lambda_{N}(E)} .
$$


By integrating Eq. (3) we obtain the nonlinear integral equation

$$
Z_{N N}^{ \pm}(E, h)=\frac{1}{h} \int_{0}^{h} d t \int_{0}^{1} d x \Phi_{N N}^{ \pm}(E, x) \eta_{N N}^{ \pm}(E, x) \exp \left[-t D_{N N}^{ \pm}(E, x, t)\right],
$$

which can be solved by iterations. The simple choice of zero-order approximation is $Z_{N N}^{ \pm(0)}(E, h)=0$, that is $D_{N N}^{ \pm(0)}(E, x, h)=1 / \lambda_{N}(E / x)-1 / \lambda_{N}(E)$. For the $n$-th step we find

$$
Z_{N N}^{ \pm(n)}(E, h)=\frac{1}{h} \int_{0}^{h} d t \int_{0}^{1} d x \Phi_{N N}^{ \pm}(E, x) \eta_{N N}^{ \pm}(E, x) \exp \left[-t D_{N N}^{ \pm(n-1)}(E, x, t)\right]
$$

where

$$
D_{N N}^{ \pm(n-1)}(E, x, h)=\frac{1-Z_{N N}^{ \pm(n-1)}(E / x, h)}{\lambda_{N}(E / x)}-\frac{1-Z_{N N}^{ \pm(n-1)}(E, h)}{\lambda_{N}(E)} .
$$

Nontrivial structure of the nucleon $Z$-factors (Fig. 1) results from the non-power law behavior of the ATIC-2 primary spectrum 24 , non-scaling behavior of the particle production cross-sections and the energy dependence of inelastic hadron-nucleus cross-sections. After that, using the obtained nucleon fluxes, we are able to calculate successively the meson and the lepton fluxes (see Ref. 16 for details).
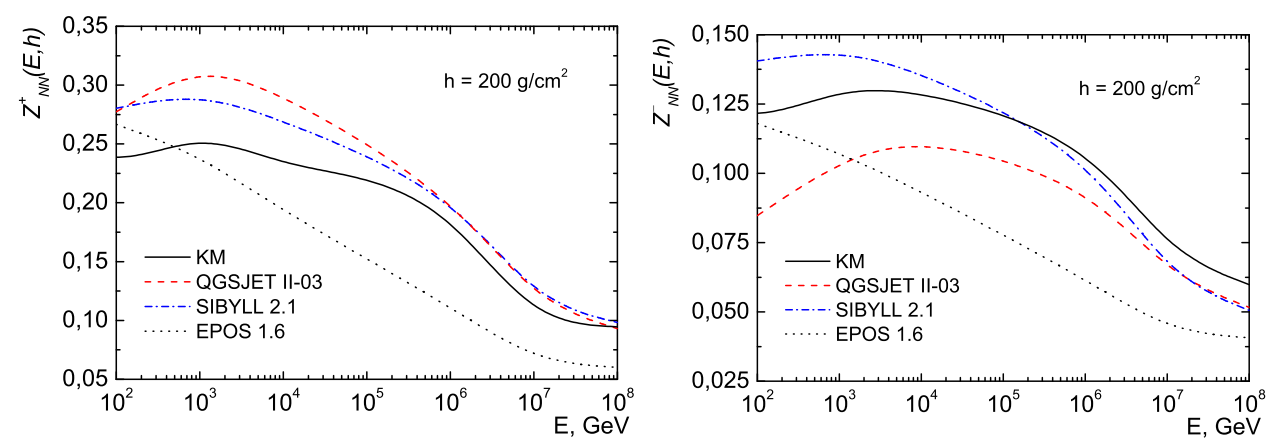

Fig. 1. The energy dependence of the nucleon $Z_{N N}^{+}(E, h)$-function (left) and $Z_{N N}^{-}(E, h)$ one (right) at the atmosphere depth of $200 \mathrm{~g} \cdot \mathrm{cm}^{-2}$ calculated for the ATIC-2 primary spectrum.

In our calculations we rely on recent data on the primary cosmic ray (PCR) spectra and composition obtained with Advanced Thin Ionization Calorimeter in the balloon-borne experiment ATIC-2 24. In order to extend the calculations to higher energies, up to $10 \mathrm{PeV}$, we use the data of the GAMMA experiment 25 . The energy spectra and elemental composition, obtained in the GAMMA experiment, cover the $10^{3}-10^{5} \mathrm{TeV}$ range and agree with the corresponding extrapolations of known balloon and satellite data at $E \geq 10^{3} \mathrm{TeV}$. Alternative primary spectra, used in the calculations for a region of very high energies, is the model by Zatsepin and Sokolskaya $(\mathrm{ZS})$ 26 27. The ZS proton spectrum at $E \gtrsim 10^{6} \mathrm{GeV}$ is compatible with 
KASCADE data 28129 and the helium one is within the range of the KASCADE spectrum recontructed with use of QGSJET 01 and SIBYLL models. Besides we use also the Gaisser and Honda spectra ${ }^{30}(\mathrm{GH})$ to compute the muon flux in more narrow energy range $E_{\mu} \lesssim 10^{5} \mathrm{GeV}$.

\section{High-energy muon spectra}

Apart from evident sources of AM, $\pi_{\mu 2}$ and $K_{\mu 2}$ decays, we take into consideration three-particle semileptonic decays, $K_{\mu 3}^{ \pm}, K_{\mu 3}^{0}$. Also we take into account small fraction of the muon flux originated from decay chains $K \rightarrow \pi \rightarrow \mu \quad\left(K_{S}^{0} \rightarrow \pi^{+}+\pi^{-}\right.$, $\left.K^{ \pm} \rightarrow \pi^{ \pm}+\pi^{0}\right)$.

The high energy spectra of the conventional and prompt muons at ground level calculated for the vertical direction are shown in Fig. 2 together with the experimental data. The inclined shaded bands here indicate the conventional muon flux calculated with KM model for the case of the ATIC-2 primary spectrum (narrow light band on the left) and the GAMMA one (dark band). The size of the bands corresponds to statistical errors in the ATIC-2 and GAMMA experiments. Solid, dashed, dash-dotted, and dotted lines indicate the calculations with use of ZS spectrum and set of hadronic models, KM, SIBYLL 2.1, EPOS 1.61, and QGSJET-II 03. The experimental data comprise the measurements of L3+Cosmic 31 , CosmoALEPH $32 \mid 33$ as well as the data (converted to the surface) of deep underground experiments MSU $\stackrel{34}{ }$, MACRO $\stackrel{35}{ }$, LVD $\stackrel{36}{ }$, Frejus 37 , Baksan $\frac{38}{38}$, Artyomovsk 39 . Notice that the calculation results do not fit well the Frejus and MSU data even if the prompt muon component is taken into account (thin lines 1-4 in Fig. 2), while the LVD data are well described.

The ZS model seems to be a reasonable bridge from $\mathrm{TeV}$ energy range to $\mathrm{PeV}$ one (solid line), providing a junction of the different energy ranges. However, above $10^{6} \mathrm{GeV}$ the muon flux is apparently affected by the primary cosmic ray ambiguity in the vicinity of 'knee'. To illustrate this we plot also our early predictions $18 / 40 \mid 41$ for the conventional muon flux made with primary cosmic ray spectra by Nikolsky, Stamenov, and Ushev (NSU) ${ }^{42}$ (thin line 5) as well as by Erlykin, Krutikova, and Shabelsky (EKS) 43 (dashed line 6 over the GAMMA band). The index $\gamma$ of the NSU primary nucleons is 1.62 and 2.02 before and beyond the "knee" $(\sim 3 \mathrm{PeV})$ correspondingly, while $\gamma=1.7$ and 2.1 for the EKS spectrum.

The prompt muon contribution due to decays of charmed hadrons at high energies is shown here for several charm production models: the recombination quarkparton mode ${ }^{17}$ (RQPM, line 1 in Fig. 2), the two calculation versions of the model

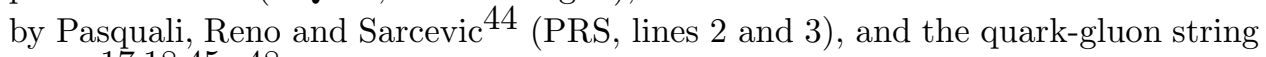
mode 17/18/45-48 (QGSM, line 4). Besides we draw the shaded band (below the line 4) calculated in Ref. 22 that displays the theoretical uncertainty for the prompt muon flux due to the QCD dipole mode $22(\mathrm{DM})$. In this case we consider the prompt muon neutrino flux to be equal to the prompt muon one. Also here we ignore the differences in prompt muon flux calculations related both to atmospheric 
hadron cascade features and the primary cosmic ray spectrum and composition. Both RQPM and QGSM are the nonperturbative models, whereas PRS 44 as well as $\mathrm{DM}^{22}$ are based on the next-to-leading-order $\mathrm{QCD}$ calculations. At the muon energy above $2 \mathrm{PeV}$ the upper edge of the DM theoretical uncertainty band is in close agreement with the QGSM prediction $17 \mid 18$.

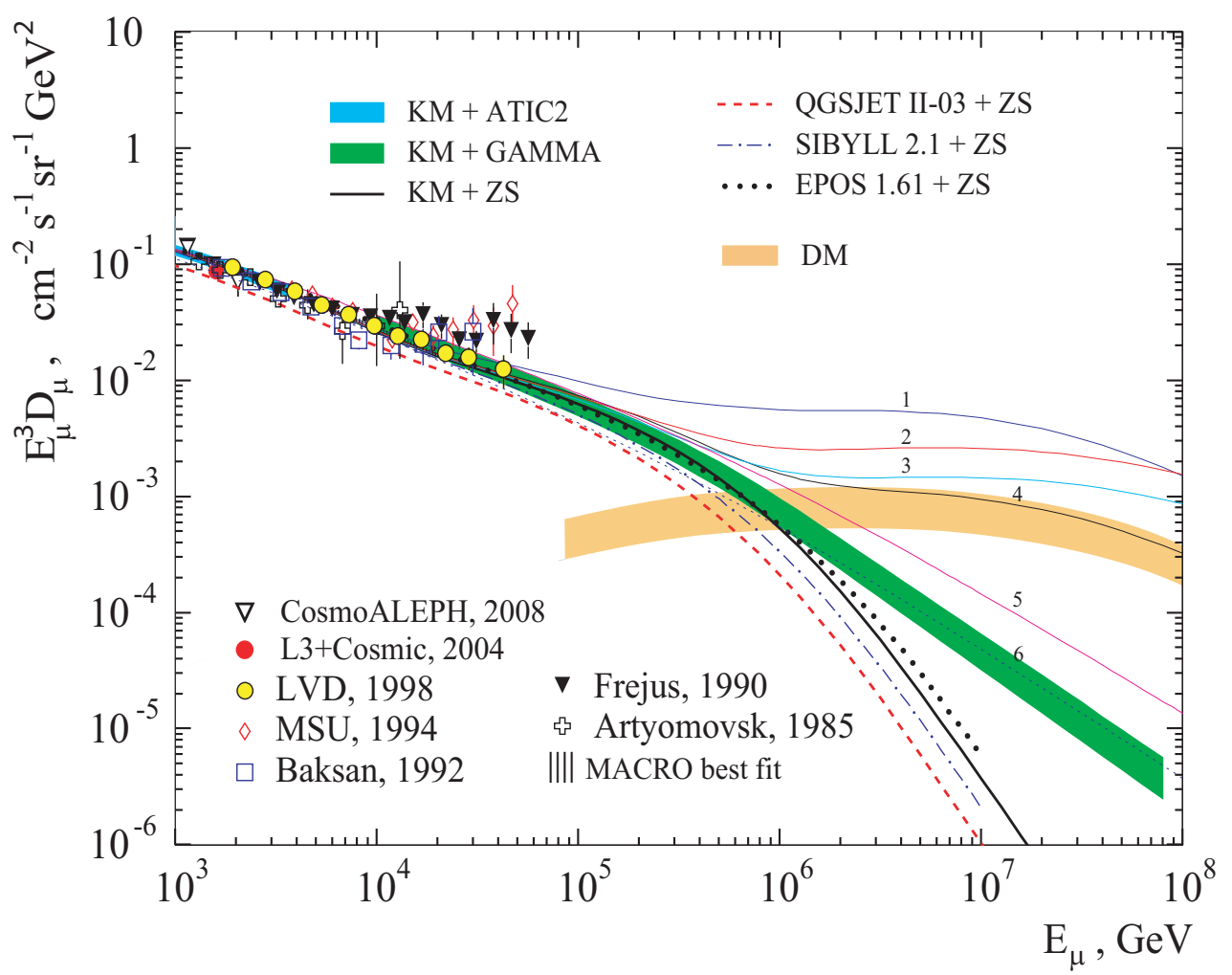

Fig. 2. The high energy vertical muon spectra at ground level. The dashed-line curves and inclined shaded areas present this work calculations for the KM mode 14 with the ATIC-2 primary spectrum $24\left(E_{\mu}<10 \mathrm{TeV}\right)$ and GAMMA one $\left(E_{\mu}>10 \mathrm{TeV}\right)$. The solid curve marks the computation for the primary spectrum model by Zatsepin and Sokolskayd $26 \mid 27$. The numbers near thin lines indicate the prompt muon flux model $(1-4)$ and the conventional muon flux $(5,6): 1$ - RQPM 17,2 and 3 - two calculation versions of PRS mode 14,4 - QGSM17]18, 5 - KM model combined with NSU cosmic ray spectrum $\frac{42}{2}, 6$ - the same with EKS spectrum 43 . The shaded band (DM) below the line 4 presents the prompt neutrino flux calculation of Ref 22

\section{Muon charge ratio}

The muon charge ratio depends on the proton to neutron ratio in primary cosmic rays as well as on the hadron production cross-sections. Thus, a comparison of the calculated $\mu^{+} / \mu^{-}$ratio with experimental data in a wide energy range gives 
a possibility to study indirectly these features. At present time the muon charge ratio is measured with new facilities, that supply with high quality data from large number of muon events at high energies.

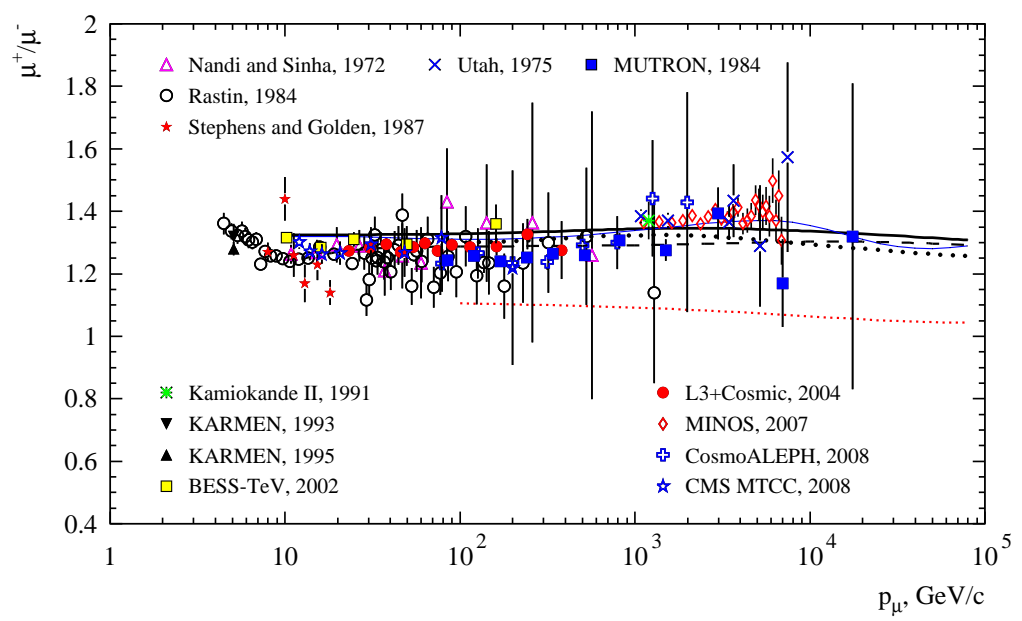

Fig. 3. Muon charge ratio at ground level computed for the three hadronic interaction models and the two primary cosmic ray spectra. Solid line marks the $\mathrm{KM}+\mathrm{GH}$ result for $\theta=0^{\circ}$, dashed line shows the same at $90^{\circ}$. Thin line: the $\mathrm{KM}+\mathrm{ZS}$ at $0^{\circ}$, bold-dotted: the SIBYLL $2.1+\mathrm{GH}$, dotted (the lower): the QGSJET-II + GH at $0^{\circ}$.

In Fig. 3 we present our calculations of $\mu^{+} / \mu^{-}$ratio along with the data of experiments $31 / 3349$ 58. The calculations are made with the three hadronic interaction models and the two primary cosmic ray spectra. Solid line and dashed one mark the $\mathrm{KM}+\mathrm{GH}$ computation for the zenith angles $0^{\circ}$ and $90^{\circ}$, thin line indicates the $\mathrm{KM}+\mathrm{ZS}$ result at $0^{\circ}$. Bold dotted line indicates the SIBYLL $2.1+\mathrm{GH}$ result, dotted shows the QGSJET-II + GH one, both for $0^{\circ}$. One may see that the KM and SIBYLL calculations reproduce data well with both versions of the primary spectra, GH and ZS. The calculated curves correspond approximately to the value 1.3, that is in agreement with the recent data of BESS-TeV, CosmoALEPH, and L3+Cosmic experiments up to $1 \mathrm{TeV}$. For higher energies the kaon source of muons becomes more intensive leading to a maximum of the muon ratio, $\sim 1.4$, at energy close to $10 \mathrm{TeV}$. This value agrees with the recent results of the MINOS far detector 56 . The calculations also are in agreement with the spectrograph MUTRON data 52 at $\theta=89^{\circ}$ including the point above $10 \mathrm{TeV}$.

The QGSJET-II model (lower line) shows visible deviation from others: the predicted $\mu^{+} / \mu^{-}$ratio is close to $\sim 1.2$, that might be explained by the higher extent of the proton and neutron flux equalization in the atmosphere due to reactions $p A \rightarrow n X$ in the model (see Fig. 1). 


\section{Summary}

The conventional muon spectrum in the energy region $0.1-100 \mathrm{PeV}$ is calculated with usage of known hadronic models, QGSJET-II, SIBYLL 2.1, EPOS and KM, basing on experimental measurements of the primary cosmic ray spectrum and taking into account the "knee". We study the slope influence on tne muon spectrum in wide energy region up to the muon energy $100 \mathrm{PeV}$, far beyond the knee.

The calculation around the knee region (Fig.2) reveals strong dependence of the conventional muon flux on the hadronic models (involving $u, d, s$ quarks). Uncertainty in the flux due to the hadronic models reaches a half of order of magnitude, that is comparable with the prompt muon flux uncertainty related to differences among charm production models, RQPM and QGSM or DM, for example. One may suppose that the primary cosmic ray uncertainty beyond the knee, as well as the hadronic model uncertainty, would be negligible provided that real prompt muon flux at the energy around $\sim 10^{6} \mathrm{GeV}$ is not too different from the QGSM prediction (Fig. 2).

It seems reasonable to consider that the prompt muon flux higher than it is predicted by RQPM to be excluded 3 . One may expect that more strong restriction of the prompt muon flux range will be extracted from the experiment in the near future.

\section{Acknowledgements}

This research was supported in part by the Russian Federation Ministry of Education and Science within the program "Development of Scientific Potential in Higher Schools" under grants 2.2.1.1/1483, 2.1.1/1539, the Federal Target Program "Scientific and educational specialists for innovative Russia", contract No. P1242.

\section{References}

1. V. Aynutdinov et al., Astropart. Phys. 25, 140 (2006).

2. V. Aynutdinov et al., Nucl. Instrum. Meth. A 588, 99 (2008).

3. A. Achterberg et al. (IceCube Collaboration), Phys. Rev. D 76, 042008 (2007).

4. M. Ackermann et al., Astrophys. J. 675, 1014 (2008).

5. P. Berghaus for the IceCube Collaboration, Nucl. Phys. B (Proc. Suppl.) 196, 261 (2009).

6. P. Berghaus for the IceCube Collaboration, arXiv:0909.0679v1.

7. A. A. Kochanov, T. S. Sinegovskaya, S. I. Sinegovsky, axXiv:0906.0671v2.

8. S. S. Ostapchenko, Nucl. Phys. B (Proc. Suppl.) 151, 143 (2006).

9. S. Ostapchenko, Phys. Rev. D 74, 014026 (2006).

10. R. S. Fletcher et al., Phys. Rev. D 50, 5710 (1994).

11. R. Engel et al. in Proc. 26th Int. Cosmic Ray Conf., Salt Lake City, 1999, vol. 1, p. 415 .

12. K. Werner, T. Pierog, AIP Conf. Proc. 928, 111 (2007).

13. K. Werner, Nucl. Phys. B (Proc. Suppl.) 175-176, 81 (2008).

14. A. N. Kalinovsky, N. V. Mokhov, Yu. P. Nikitin, Passage of high-energy particles through matter, AIP, New York, 1989. 
15. A. A. Kochanov et al., astro-ph/0706.4389.

16. A. A. Kochanov, T. S. Sinegovskaya, S. I. Sinegovsky, Astropart. Phys. 30, 219 (2008).

17. E. V. Bugaev et al., Nuovo Cim. C 12, 41 (1989).

18. E. V. Bugaev et al., Phys. Rev. D 58, 054001 (1998).

19. C. G. S. Costa, Astropart. Phys. 16, 193 (2001).

20. A. Misaki et al., J. Phys. G 29, 387 (2003).

21. S. Panda, S. I. Sinegovsky, Int. J. Mod. Phys. A 23, 2933 (2008).

22. R. Enberg, M. H. Reno, I. Sarcevic, Phys. Rev. D 78, 043005 (2008).

23. V. A. Naumov, T. S. Sinegovskaya, Phys. Atom. Nucl. 63, 1927 (2000).

24. A. D. Panov et al., Bull. Russ. Acad. Sci. Phys. 71, 494 (2007).

25. A. P. Garyaka et al., Astropart. Phys. 28, 169 (2007).

26. V. I. Zatsepin, N. V. Sokolskaya, Astron. Astrophys. 458, 1 (2006).

27. V. I. Zatsepin, N. V. Sokolskaya, Astron. Lett. 33, 29 (2007).

28. T. Antoni et al., Astropart. Phys. 24, 1 (2005).

29. W. D. Apel et al., Astropart. Phys. 31, 86 (2009).

30. T. K. Gaisser, M. Honda, Annu. Rev. Nucl. Part. Sci. 52, 153 (2002).

31. P. Achard et al., Phys. Lett. B 598, 15 (2004).

32. N. Omar Hashim, CERN-THESIS-2007-047.

33. C. Grupen, Nucl. Phys. B (Proc. Suppl.) 175-176, 286 (2008).

34. G. T. Zatsepin et al., Izv. Ross. Akad. Nauk. Fiz. 58, 119 (1994).

35. M. Ambrosio et al., Phys.Rev. D 52, 3793 (1995).

36. M. Aglietta et al., Phys. Rev. D 58, 092005 (1998).

37. W. Rhode, Nucl. Phys. B (Proc. Suppl.) 35, 250 (1994).

38. V. N. Bakatanov et al., Yad. Fiz. 55, 2107 (1992).

39. R. I. Enikeev et al., Yad. Fiz. 47, 1044 (1988).

40. T. S. Sinegovskaya, S. I. Sinegovsky, Phys. Rev. D 63, 096004 (2001).

41. A. A. Kochanov, T. S. Sinegovskaya, S. I. Sinegovsky, Phys. Atom. Nucl. 70, 1913 (2007).

42. S. I. Nikolsky, I. N. Stamenov, S. Z. Ushev, Sov. Phys. JETP 60, 10 (1984).

43. A. D. Erlykin, N. P. Krutikova, Yu. M. Shabelsky, Sov.J. Nucl. Phys. 45, 668 (1987).

44. L. Pasquali, M. H. Reno, I. Sarcevic, Phys. Rev. D 59, 034020 (1999).

45. A. B. Kaidalov, O. I. Piskunova, Sov. J. Nucl. Phys. 41, 816 (1985).

46. A. B. Kaidalov, O. I. Piskunova, Sov. J. Nucl. Phys. 43, 994 (1986).

47. A. B. Kaidalov, O. I. Piskunova, Z. Phys. C 30, 145 (1986).

48. O. I. Piskunova, Sov. J. Nucl. Phys. 56, 1094 (1993).

49. S. Haino et al., Phys. Lett. B 594, 35 (2004).

50. B. C. Nandi, M. S. Sinha, J. Phys. A 5, 1384 (1972).

51. G. K. Ashley, J. W. Keuffel, M. O. Larson, Phys. Rev. D 12, 20 (1975).

52. S. Matsuno et al., Phys. Rev. D 29, 1 (1984).

53. B. C. Rastin, J. Phys. G 10, 1629 (1984).

54. S. A. Stephens, R. L. Golden, Proc. 20th ICRC, Moscow, vol. 6, 1987, p. 173.

55. T. E. Jannakos, FZKA Report 5520 (Forschungszentrum Karlsruhe, 1995).

56. P. Adamson et al., Phys. Rev. D 76, 052003 (2007).

57. M. Yamada et al., Phys. Rev. D 44, 617 (1991).

58. M. Aldaya, P. Garcia-Abia, CMS NOTE-2008/016, CERN Document Server: http://cdsweb.cern.ch 